\title{
Tobacco TTG2 regulates vegetative growth and seed production via the predominant role of ARF8 in cooperation with ARF17 and ARF19
}

\author{
Jun Ge${ }^{1}$, Baoyan Li ${ }^{1,2}$, Dan Shen ${ }^{1}$, Junyi Xie ${ }^{1}$, Juying Long ${ }^{1}$ and Hansong Dong ${ }^{1 *}$
}

\begin{abstract}
Background: Plant TRANSPARENT TESTA GLABRA (TTG) proteins regulate various developmental activities via the auxin signaling pathway. Recently, we elucidated the developmental role of tobacco (Nicotiana tabacum L.) NtTTG2 in association with 12 genes that putatively encode AUXIN RESPONSIVE FACTOR (ARF) proteins, including NtARF8, NtARF17, and NtARF19. Here we show that NtTTG2 regulates tobacco growth and development by involving the NtARF8, NtARF17, and NtARF19 genes, with the NtARF8 gene playing a predominant contribution.

Results: Independent silencing of the NtARF8 gene more strongly repressed tobacco growth than silencing the NtARF17 or NtARF19 gene and more effectively eradicated the growth enhancement effect of NtTTG2 overexpression. In contrast, plant growth was not affected by silencing additional nine NtTTG2-regulated NtARF genes. In double and triple gene silencing combinations, silencing the NtARF8 gene was more effective than silencing the NtARF17 or NtARF19 gene to repress growth as well as nullify growth enhancement. Therefore, the NtARF8 predominantly cooperated with the NtARF17 and NtAFR19 of the NtTTG2 functional pathway. NtARF8 also contributed to NtTTG2-regulated seed production as concurrent NtTTG2 and NtARF8 overexpression played a synergistic role in seed production quantity, whereas concurrent silencing of both genes caused more severe seed abortion than single gene silencing. In plant cells, the NtTTG2 protein facilitated the nuclear import of NtARF8 as well as increased its function as a transcription activator.
\end{abstract}

Conclusions: NtARF8 is an integral component of the NtTTG2 functional pathway, which regulates tobacco growth and development.

\section{Background}

TRANSPARENT TESTA GLABRA (TTG) proteins are essential regulators of plant trichome and seed development $[1,2]$ and have been extensively studied in terms of the functional regulation [3-5]. TTGs are characterized by the presence of the WD40 motif, which comprises the conserved tryptophan (W) and aspartic (D) dipeptide within a length of approximately 40 amino acid residues [6-11]. With WD40, TTGs can directly interact with various types of proteins or serve as

\footnotetext{
* Correspondence: hsdong@njau.edu.cn

${ }^{1}$ Plant Growth and Defense Signaling Laboratory, State Ministry of Education Key Laboratory of Integrated Management of Crop Pathogens and Insect Pests, Nanjing Agricultural University, Nanjing 210095, China

Full list of author information is available at the end of the article
}

interchangeable substrate adaptors that influence proteinprotein interactions $[11,12]$ and therefore are capable of regulating both plant development and immunity $[4,10$, $13,14]$. Depending on the biochemical characteristics of cooperative partners, TTGs possess different functions relating to development and immune response $[4,8,10,15]$.

Tobacco (Nicotiana tabacum L.) NtTTG1 and NtTTG2 share a high similarity and four WD40 repeats $[4,10]$. The WD40 domain enables NtTTG1 to interact with elicitin protein ParA1, which is produced by an oomycete pathogen and induces hypersensitive cell death in plants [10]. The NtTTG1-ParA1 interaction is essential for the induction of programmed cell death initially in leaf trichomes and then in mesophylls, which in turn results in plant resistance to different pathogens [10]. In contrast, NtTTG2 
suppresses pathogen resistance in tobacco by indirectly modulating the subcellular localization of protein, NONINDUCER OF PATHOGENESIS-RELATED GENES1 (NPR1) [4], which is a transcription activator of immune response genes [16, 17]. NtTTG2 does not interact with NPR1, but sequesters NPR1 from the nucleus, thereby preventing NPR1 from transcriptionally regulating immune responses [4]. In another study paradigm, AtTTG1 of Arabidopsis thaliana Johannes Thal interacts with the bHLH transcription factor GL3, whereas its heterogenous binary complex further interacts with the MYB transcription factor GL1 to form a WD40-bHLH-MYB triplet, which regulates trichome development [12, 18]. These findings suggest that TTGs regulate plant development or immunity by either directly or indirectly interacting with their functional partners.

We have elucidated the developmental function of NtTTG2 by analyses of NtTTG2-overexpressing (TTG2 ${ }^{+}$) and NtTTG2 silencing (TTG2i) transgenic tobacco lines compared to wild-type (WT) plants or a transgenic control line [4]. The transgenic control line WT:RFP, which harbors the gene encoding red-fluorescent protein (RFP), resembles the WT in terms of vegetative growth, seed production, and related physiological responses such as floral anthocyanin synthesis and flower colorization [19]. In contrast, growth and development are greatly enhanced in NtTTG2-overexpressing $T T G 2^{+}: R F P$ lines [19], which accumulate the NtTTG2-RFP fusion protein in both the cytoplasm and nucleus [4]. The NtTTG2-confered traits of WT plants can be impaired by hairpin-based posttranscriptional gene silencing [4].

We further demonstrate that the NtTTG2 functional pathway for developmental regulation associates with the components of the auxin signaling pathway [19]. In particular, auxin-responsive genes have been identified by de novo assembly of the transcriptome of the WT, $T_{T G 2^{+}}$, and TTG2i plants with a common tobacco variety NC89 background following RNA-Seq analyses [19]. The tobacco transcriptome contains 303 unigenes that are related to auxin responses, including 40 unigenes that are predicted to encode AUXIN RESPONSE FACTOR (ARF) proteins [19]. The expression levels of 27 putative NtARF genes were unrelated to NtTTG2, whereas the other $13 N t A R F$ candidates were regulated by NtTTG2 at the transcriptional level [19]. These findings suggest that the function of NtTTG2 in plant development might be related to the auxin signaling pathway.

The auxin signaling pathway regulates various aspects of plant development $[20,21]$ through the function of ARFs in transcriptional regulation of auxin responses [22-26]. The regulatory consequence to developmental processes is either positive or negative as ARFs may activate or repress auxin-responsive genes [27-29]. In Arabidopsis, for example, 23 ARFs are categorized either as transcription activators or repressors [30]. ARF transcription activators are characterized by the presence of several glutamine $(\mathrm{Q})$ residues in the middle region of protein sequence, whereas transcription repressors are serine-rich in the same region [27, 31]. These ARFs target auxin-responsive genes of the Small Auxin Up RNA (SAUR), Auxin/Indole-Acetic Acid inducible (AUX/IAA), and Gretchen Hagen 3 (GH3) gene families [31-33]. Different ARFs regulate the expression of target genes by binding the auxin response elements, TGTCTC, GAGACA $[29,34,35]$, or TGTCT [26] that are present in target promoters. These elements have numerous potential combinations with ARFs $[4,19,30]$, but only a few auxinresponsive genes have been shown to be targeted and directly regulated by a specific NtARF $[26,31]$. Moreover, ARFs are functionally coregulated by various combinations of AUX/IAA proteins [30, 36]. In essence, despite the large number of ARFs characterized in Arabidopsis and other species, direct targets of ARFs and the individual and combinatorial roles remain largely unknown, particularly in species other than Arabidopsis.

The purpose of the present study was to identify $N t A R F$ genes that are associated with the developmental role of NtTTG2, and to elucidate the functional relationship between NtTTG2 and relevant NtARFs in tobacco. We focused on $13 N t A R F$ candidates that have been previously demonstrated to be regulated by NtTTG2 for expression in tobacco [19]. We followed the accepted nomenclature in designating $N t A R F$ gene candidates as number-suffixed $A R F \mathrm{~s}$ based on the highest similarities with the corresponding orthologs of different plant species.

Previously, a posttranscriptional gene silencing (VIGS) system was developed with DNA components of the monopartite Begomovirus sp. tobacco curly shoot virus [37]. We have used the TCSV VIGS system to silence NtTTG1 [10] and immunity-regulatory genes [38] in tobacco. In the present study, we employed the same system to silence the NtARF genes under backgrounds of WT:RFP, TTG2 ${ }^{+}: R F P$, and TTG2i. Based on the gene silencing effects, we propose that NtTTG2 predominantly regulates NtARF8, followed by NtARF17 and NtARF19, to control vegetative growth and seed production in tobacco.

\section{Results}

NtTTG2 responds to auxin but does not affect endogenous auxin levels

To infer the functional relationship between NtTTG2 and auxin, we analyzed NtTTG2 expression in leaves of WT tobacco treated with an aqueous solution containing a surfactant and a synthetic auxin, 1-naphthaleneacetic acid (NAA), and with the surfactant solution as control. The NAA treatment highly induced NtTTG2 expression based on quantitative real-time RT-PCR (RT-qPCR) analysis performed at a 10-min interval in $60 \mathrm{~h}$ after plant treatment 
(Fig. 1, curves). In the period, quantities of NtTTG2 transcript were increased by 4-6-fold following the NAA treatment compared to the steady-state transcript level of the control (Fig. 1, curves). Marked enhancement of NtTTG2 expression was confirmed by Northern blotting that was performed at $60 \mathrm{~h}$ after plant treatment. In Northern blotting, the NtTTG2 transcript was detected at a steady-state level compared to the constitutively expressed EF1 $\alpha$ gene in control plants, whereas NtTTG2 was upregulated after NAA treatment (Fig. 1, inset).

We measured the endogenous concentrations of IAA in leaves of WT:RFP, TTG2 $2^{+}$RFP, and TTG2i plants. The $T T G 2^{+}: R F P$ line showed the best developmental traits with the highest level of TTG2 overexpression, and the TTG2i line displayed the highest degrees of NtTTG2 silencing and developmental compromise [4, 19]. No distinct differences in endogenous IAA content in the aerial organs were observed among the three tobacco genotypes were observed. In all plants, IAA concentrations were detected at basal levels of approximately 70, 150, and $230 \mathrm{ng} / \mathrm{g}$ fresh weight of leaves, flowers, and immature fruits (capsules), respectively (Additional file 1: Figure S1). Therefore, NtTTG2 is responsive to exogenous auxin application, whereas no effect was observed using endogenous auxin content. Because NtARFs are essential for auxin signaling and function of downstream processes of auxin biosynthesis [20,31], we considered NtTTG2-regulated NtARF candidates [19] with respect to the functional connection between NtTTG2 and auxin signaling.

\section{The 13 NtTTG2-regulated NtARF candidates represent 12 NtARF genes}

According to conventional nomenclature of unigenes identified by the RNA-Seq technique [39], NtTTG2regulated NtARF candidates present in the tobacco transcriptome were specified with unigene codes [19]. For the sake of convenient descriptions, we designated NtARF unigenes as conventional gene symbols, with NtARF suffixed by numbers. Based on the highest similarities of unigenes with NtARF orthologs previously identified in different plant species, the 13 NtTTG2-regelated NtARF unigenes represented $12 N t A R F$ genes because two unigenes were classified under the same NtARF gene (Additional file 2: Table S1). Sequence similarities were considered with tobacco species as first priority over other plants such as Arabidopsis and tomato (Solanum lycopersicum L.). The 12 NtTTG2-regulated tobacco NtARF genes were thus designated as NtARF1, NtARF2, NtARF5, NtARF6L (NtARF6-Like), NtARF8, NtARF9, NtARF11, NtARF16 to NtARF19, and NtARF19L (NtARF19-like) (Additional file 2: Table S1). Similarities in nucleotide

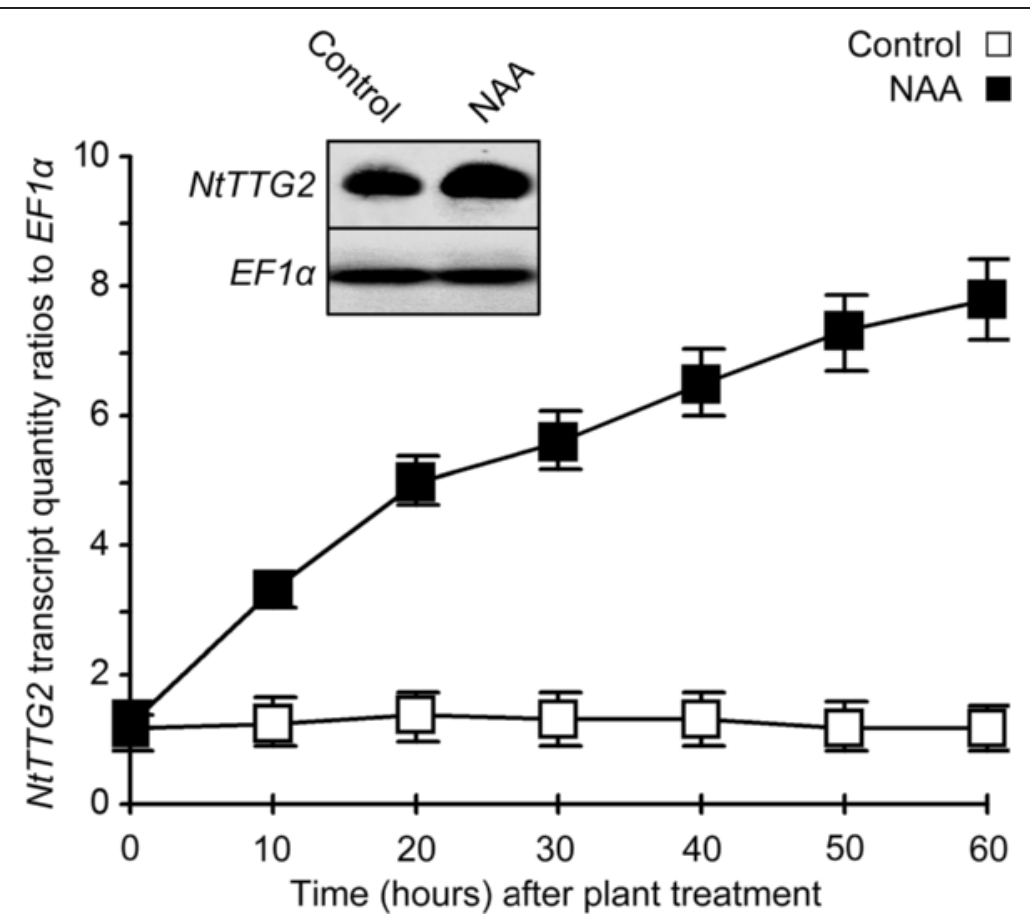

Fig. 1 The expression of NtTTG2 in response to synthetic auxin NAA. Plants were treated by spraying over tops with a surfactant solution in control or with an NAA solution containing the surfactant. Relative levels of NtTTG2 expression were quantified by RT-qPCR as the transcript quantity ratios to the constitutively expressed EFla gene used as a reference. Data shown are mean values \pm standard errors from mean (SEMs) of results obtained from three experimental replicates, i.e., the number of replicates $(n)=3$. Inset is Northern blot hybridization performed at $60 \mathrm{~h}$ after plant treatment 
sequences were easily compared with published $A R F$ homologs by using BLAST.

\section{The 12 NtARFs fall into NtTTG2-upregulated and -down- regulated groups}

To elucidate the functional relationship between NtTTG2 and NtARF genes, the 12 NtTTG2-regulated $N t A R F \mathrm{~s}$ were silenced under backgrounds of WT:RFP, $T T G 2^{+}$, and TTG2i, followed by assessments of gene expression levels in leaves of distinct plant genotypes. The VIGS system previously developed with DNA components of TCSV [37] and recently used by our group to silence NtARF8 [38] was employed in the present study to construct silencing units of an additional 11 $N t A R F$ genes (Fig. 2a). In parallel experiments, NtARF gene silencing constructs was used to transfect tobacco plants, followed by verification of transgene integration into the plant genome (Fig. 2b). Then, relative levels of $N t A R F$ gene expression were quantified by RT-qPCR, which varied with the genetic background of each plant (Fig. 2c).

The elaborate experimental protocol resulted in high gene silencing efficiencies, as shown by marked decreases in the quantities of the corresponding gene transcripts as detected by semi-quantitative RT-PCR (Fig. 2b) and RT-qPCR (Fig. 2c) analyses. All 12 NtTTG2-regulated NtARF genes were silenced at high efficiencies $(>95 \%)$ compared to the steady-state expression levels under WT:RFP background (Fig. 2c). Under the TTG2 $2^{+}$RFP background, NtARF6L and NtARF19L had fewer transcripts, but the additional ten genes (NtARF1, NtARF2, NtARF5, NtARF8, NtARF9, NtARF11, NtARF16, NtARF17, NtARF18 and NtARF19) accumulated more transcripts than the WT:RFP plant. These genes showed opposite behaviors under a TTG2i background compared to TTG2 $2^{+}$RFP (Fig. 2c). Thus, NtARF1, NtARF2, NtARF5, NtARF8, NtARF9, NtARF11, NtARF16, NtARF17, NtARF18 and NtARF19 comprised a group of NtTTG2-upregulated NtARF genes, whereas NtARF6L and NtARF19L represented the downregulated group. In the ten NtTTG2-upregulated NtARFs, NtARF8 displayed the highest degree of expression enhancement by NtTTG2 (Fig. 2c). On the basis of downregulation by TTG2i, expression levels of NtTTG2upregulated $N t A R F$ s were further reduced in double gene silencing TTG2i/ARFi lines. Inversely, NtARF expression levels were partially recovered in $T T G 2^{+}: R F P / A R F i$ plants, which resulted from the silencing NtARFs under a TTG2 ${ }^{+}$:RFP background (Fig. 2c). The high levels of single $N t A R F$ gene silencing and the synergistic effects of NtTTG2 and NtARF double modifications of NtARF expression may be further investigated in future studies to elucidate the roles of NtARFs in NtTTG2-regulated plant growth and development.

\section{NtARF8, NtARF17, and NtARF19 contribute to NtTTG2-} regulated plant growth

Recently we characterized the role of NtTTG2 in tobacco growth based on increases of the biomass (fresh weight) of plants grown in a medium and in pots, respectively [19]. The contributions of the different NtARF genes to NtTTG2-regulated tobacco growth were evaluated by direct observation of growth rates (Fig. 3) and by comparing fresh weight changes (Fig. 4) of the different plants at 15 days after transfection. No apparent differences in growth rates between WT:RFP and WT were observed, neither between $T T G 2^{+}$and $T T G 2^{+}: R F P$ plants (Fig. 3a compared to Fig. 3b), thereby confirming the absence of any phenotypic effects in the controls. The potential effects of NtARFs on NtTTG2-regulated plant growth were assessed by imaging plants with and without NtARF gene silencing manipulations (Fig. $3 \mathrm{c}-\mathrm{n}$ compared to Fig. 3b). The low degree of plant growth repression was caused by silencing NtARF1, NtARF2, NtARF5, NtARF6L, and NtARF9, respectively (Fig. 3c-f, h compared to Fig. 3b). A marked repression of growth was observed with $N t A R F 8, N t A R F 17$, or NtARF19 silencing. Independent silencing of the three $N t A R F$ s caused a marked reduction in plant size under the WT:RFP background, further reduction in TTG2i plant size, and a decrease in plant growth enhancement by $T T G 2^{+}$(Fig. $3 g$, k, and $\mathrm{m}$ compared to Fig. 3b). In contrast, silencing NtARF11, NtARF16, $N t A R F 18$, or NtARF19L did not cause evident changes in plant growth rates under the same genetic background (WT:RFP, TTG $2^{+}:$RFP, or TTG2i; Fig. $3 \mathrm{i}, \mathrm{j}, \mathrm{l}$, and n compared to Fig. $3 \mathrm{~b})$.

The observed differences in plant growth extents were confirmed by quantifying fold-changes in fresh weight of transfected plants compared to control plants (Fig. 4). Similar weight changes $(\sim 1.7$-fold $)$ were observed in $T T G 2^{+}$and $T T G 2^{+}$:RFP plants, whereas the weight of WT:RFP plants showed minimal change compared to that of the WT (Fig. 4a). These plants were used as basis in assessing the effects of NtARF silencing under the corresponding genetic backgrounds (Fig. 4b-m). A less than 0.5 -fold decrease or no evident changes were observed with silencing of NtARF1, NtARF2, NtARF5, NtARF6L, NtARF9, NtARF11, NtARF16, NtARF18 or NtARF19L under the WT:RFP background (Fig. 4b-e, $\mathrm{g}-\mathrm{k}$, and $\mathrm{m}$ compared to Fig. 4a). Similar results were obtained with silencing any of these $N t A R F$ genes under the backgrounds of $T T G 2^{+}$(or TTG2 $2^{+}: R F P$ ) and TTG2i (Fig. 4b-e, g-k, and $\mathrm{m}$ compared to Fig. 4a). In contrast, plant weight showed a 3.6-fold, 2.8-fold, and 3.1fold decrease, respectively, when NtARF8, NtARF17, and NtARF19 were silenced under the WT:RFP background (Fig. 4f, j, 1). Moreover, a 2-fold decrease in plant weight was observed after the independent silencing of NtARF8, NtARF17, and NtARF19 under the 

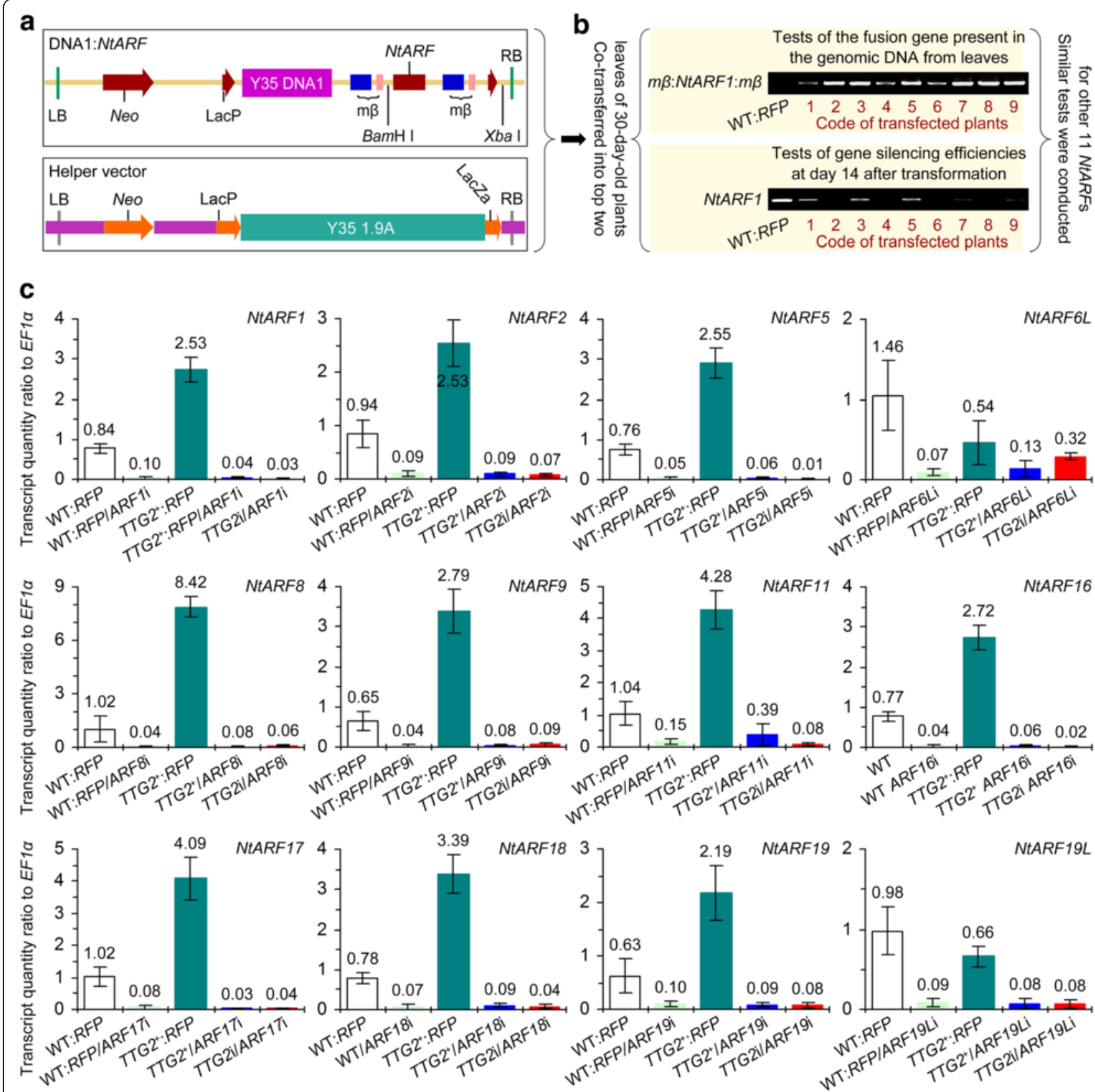

Fig. 2 VIGS manipulations of NtARF genes and gene silencing efficiencies. a Diagrams of the two-component gene-silencing constructs. b Technical scheme. The two gel panels represent one of six experimental repeats each containing nine transfected WT:RFP plants tested in comparison with WT:RFP. Top gel panel is the result of PCR analysis of genomic DNA, and lower gel panel is the result of RT-PCR analysis. Codes of transfected plants are arbitrary. c Relative levels of different NtARFs in leaves of plants indicated under the corresponding bar graphs. The expression level of each NtARF was quantified by RT-qPCR as the transcript quantity ratio of the NtARF gene to EFla used as a reference gene based on its constitutive expression. Data shown are mean values \pm SEM bars ( $n=6$ experimental replicates)

TTG2i background (Fig. 4, f, j, l) compared to that observed under WT:RFP (Fig. 4a). When NtARF17 and NtARF19 were silenced under a TTG2 $2^{+}$background, the extent of TTG2 ${ }^{+}$-conferred plant growth enhancement was reduced by 0.53 -fold (53 \%) and 1.5 -fold, respectively (Fig. 4, j and 1 compared to Fig. 4a). A stronger impairment to $T T G 2^{+}$plant growth was observed with NtARF8 silencing, which totally cancelled the growth enhancement effect and further decreased fresh weight by 1.3 -fold (Fig. 4f compared to Fig. 4a). These findings suggest that NtARF8, NtARF17, and NtARF19 are major coregulators of the NtTTG2 functional pathway for the plant growth, whereas NtARF8 plays a predominant role. 


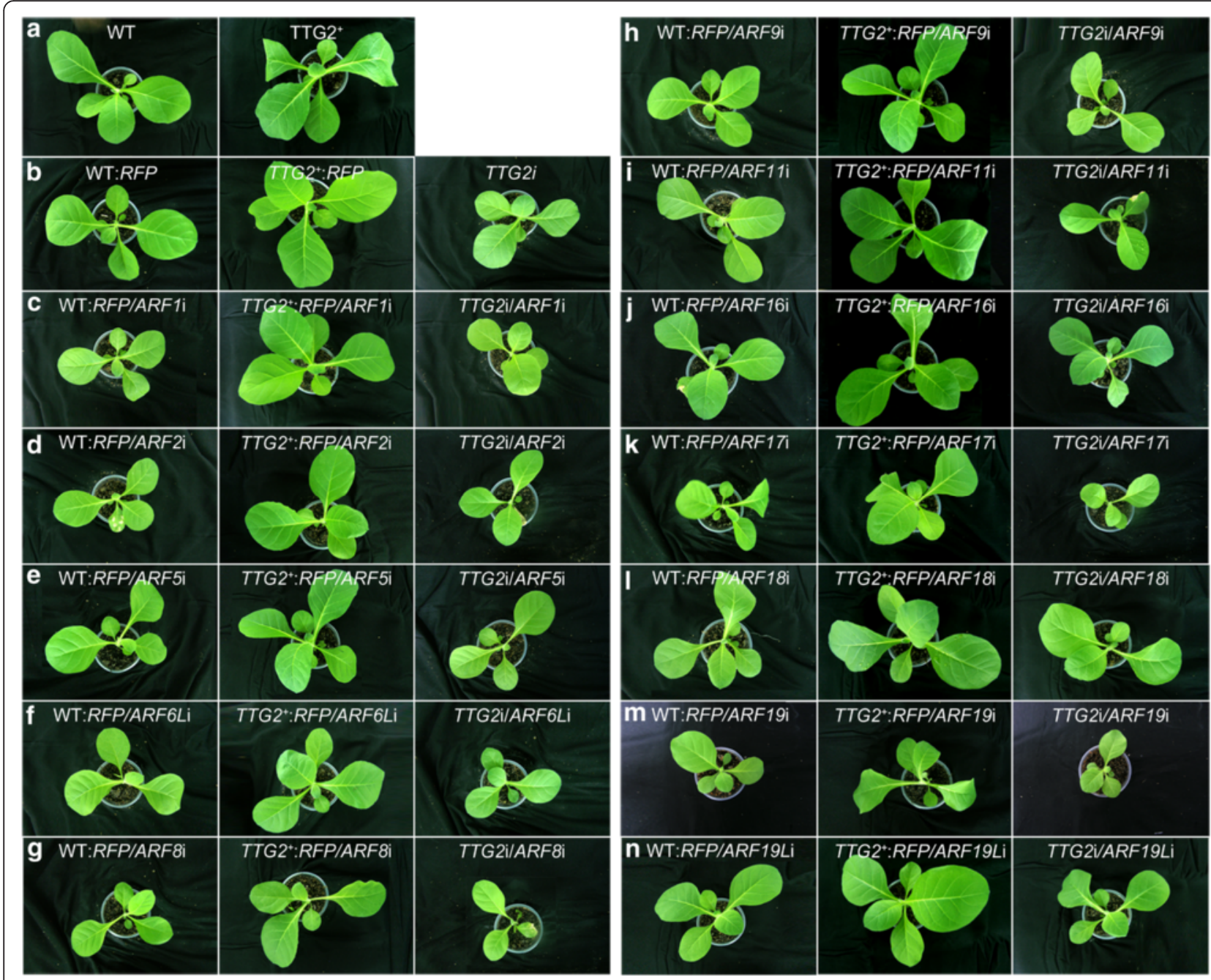

Fig. 3 Plants photographed two weeks after transfection for gene silencing manipulations. WT (or WT:RFP), TTG2 (or TTG2 :RFP), and TTG2i plants $(\mathbf{a}, \mathbf{b})$ were used as backgrounds for comparisons of the phenotypic effects of single NtARF gene silencing and concomitant silencing of TTG2 and an NtARF gene (b-n)

NtARF8 is the predominant interactor of NtARF17 and NtARF19 in NtTTG2-regulated plant growth

To confirm the roles of NtARF8, NtARF17, and NtARF19 in the NtTTG2 functional pathway, we investigated changes in fresh weight of TTG $2^{+}$plants following transfection of different leaves with each of the three $N t A R F$ VIGS constructs and with double and triple combinations at various time points. In all transfected plants, the three genes were silenced by $>95 \%$ and in an independent manner (Fig. 5a). For example, silencing $N t A R F 8$ did not affect the expression levels of NtARF17 and NtARF19, whereas concurrent silencing of NtARF8 and NtARF17 did not affect NtARF19 expression. In addition, the expression levels of NtARF8, NtARF17, and NtARF19 were simultaneously downregulated only with the triple gene silencing manipulation in single plants (Fig. 5a).
Plant weight was determined at 14 days after the last round of transfection. Based on fold-changes in fresh weight in NtARF-silenced TTG2 $2^{+}$lines compared to the WT plant, double or triple gene silencing manipulations acted synergistically in impairing $T T G 2^{+}$-conferred plant growth enhancement after single gene silencing (Fig. 5b). The most extensive compromise in plant growth enhancement was observed after silencing NtARF8 under double or triple gene silencing conditions. In double combinations, NtARF8 silencing further reduced plant weight by 1.9- and 4.9-fold based on independent reductions by NtARF17 and AFR19 silencing. However, silencing of NtARF17 and NtARF19 concurrently with $N T A R F 8$ caused an additional $25 \%$ and $38 \%$ decrease in plant weight compared to silencing NtARF8 alone. The triple gene silencing manipulation further reduced plant weight by $0.56-, 3.5-$, and 6.1-fold (56 \%, $350 \%$, and 


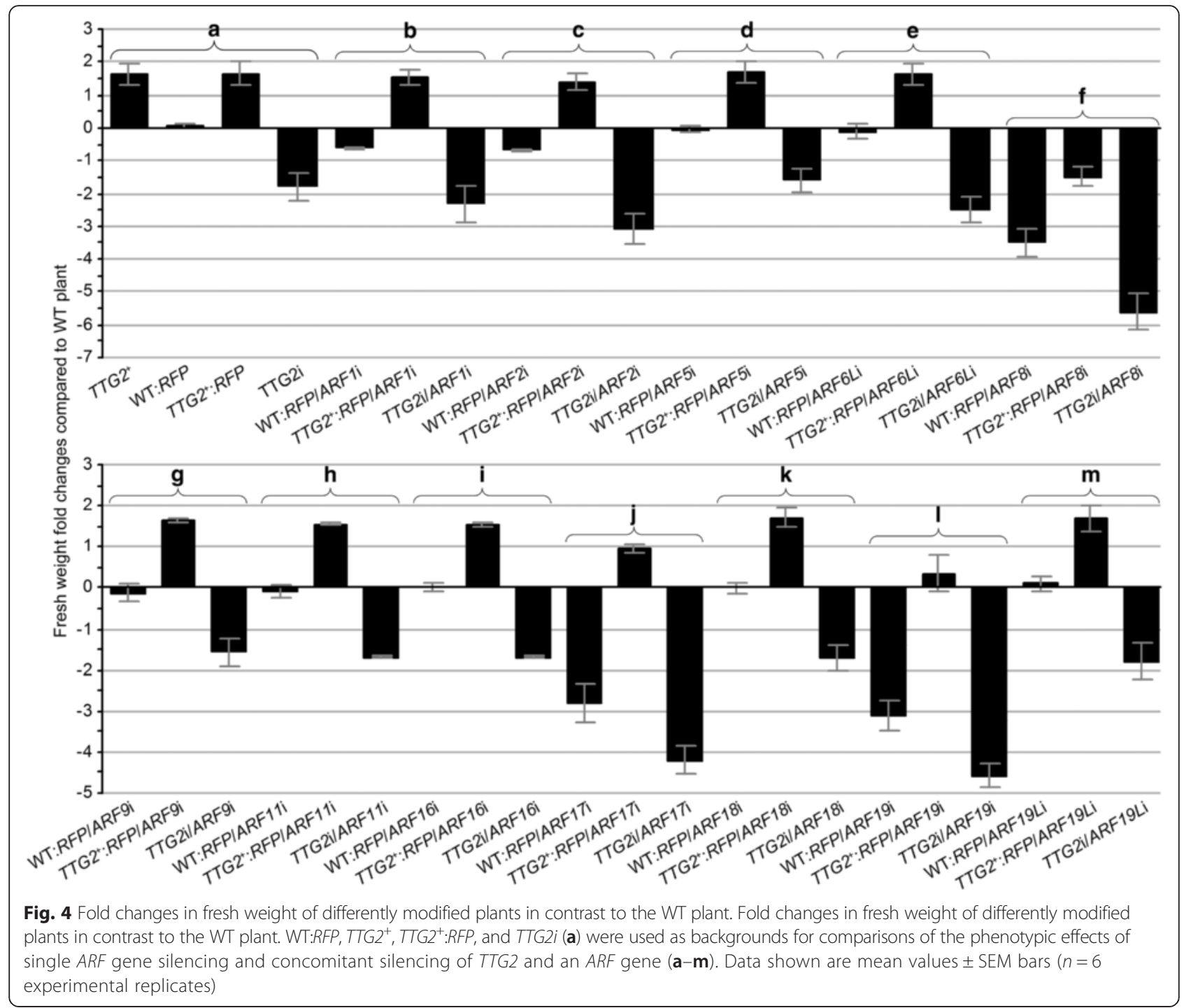

$610 \%)$ over the single gene silencing effects of NtARF8, NtARF17, and NtARF19, respectively. Clearly, silencing of NtARF8 was more repressive to plant growth than silencing NtARF17 or NtARF19. Furthermore, NtARF8 was expressed in the growing leaves of 10-50-day-old plants, much higher than that observed in NtARF17 and NtARF19 (Additional file 3: Figure S2). During plant growth, the ratios of NtARF8, NtARF17, and NtARF19 transcripts to the reference gene $(E F 1 \alpha)$ were $1.02-1.30$, $0.76-1.00$, and $0.70-0.93$, respectively. These findings suggest that NtARF8 plays a predominant role in the genetic cooperation with NtARF17 and NtARF19 in order for NtTTG2 to regulate plant growth.

NtARF8 contributes to NtTTG2-regulated seed production Similar to foliar expression (Fig. 5a), NtARF8 is also expressed in other organs, including immature fruits, in an NtTTG2-dependent manner (Additional file 4: Figure
S3). Thus, NtARF8 may participate in the NtTTG2regulated developmental process in addition to the vegetative growth. We tested this hypothesis by analyzing the functional relationship between the NtTTG2 and NtARF8 genes for tobacco seed production, which is an important developmental trait that is regulated by $N t$ TTG2 [19]. We confirmed the synergistic effect of concurrent NtTTG2 and NtARF8 silencing on the vegetative growth of elder plants (Fig. 6a-c) compared to that of younger plants (Figs. $3 \mathrm{~g}$ and $4 \mathrm{f}$ ). We also confirmed the genetic cooperation of NtTTG2 and NtARF8 by evaluating the effect of concurrent gene overexpression on plant growth (Fig. 6a-c). The transformation of WT and $T T G 2^{+}: R F P$ plants with NtARF8, which was fused to the gene encoding yellow-fluorescent protein (YFP), resulted in the transgenic lines, $A R F 8^{+}: Y F P$ and $T T G 2^{+}: R F P /$ $A R F 8^{+}: Y F P$, respectively. Five $A R F 8^{+}: Y F P$ lines and five $T T G 2^{+}: R F P / A R F 8^{+}: Y F P$ lines were characterized based on 


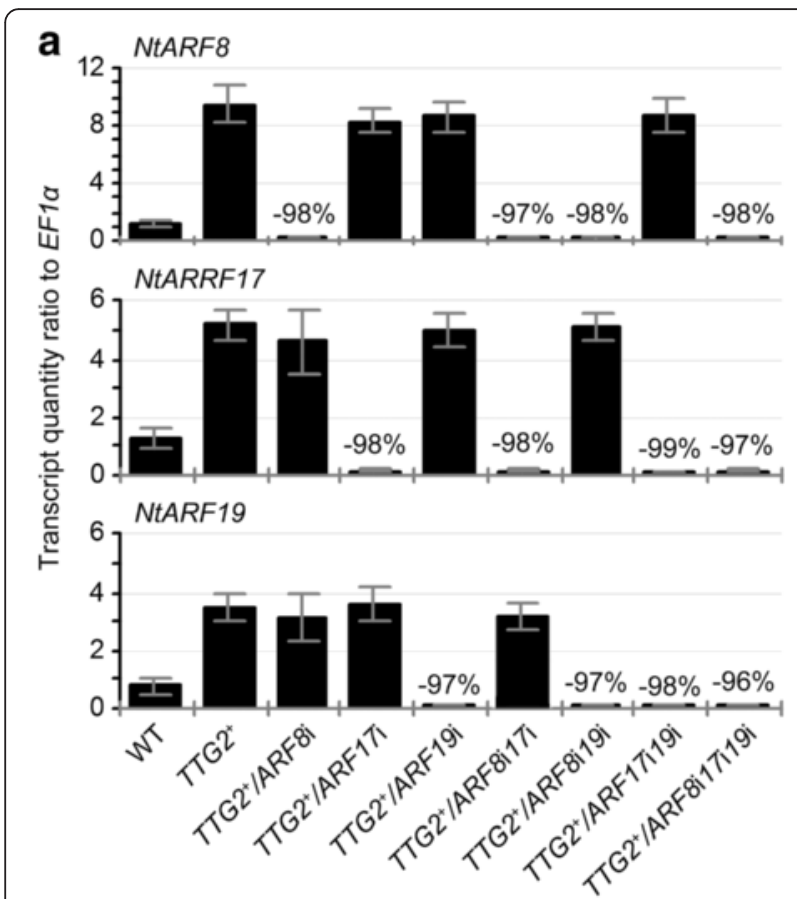

b

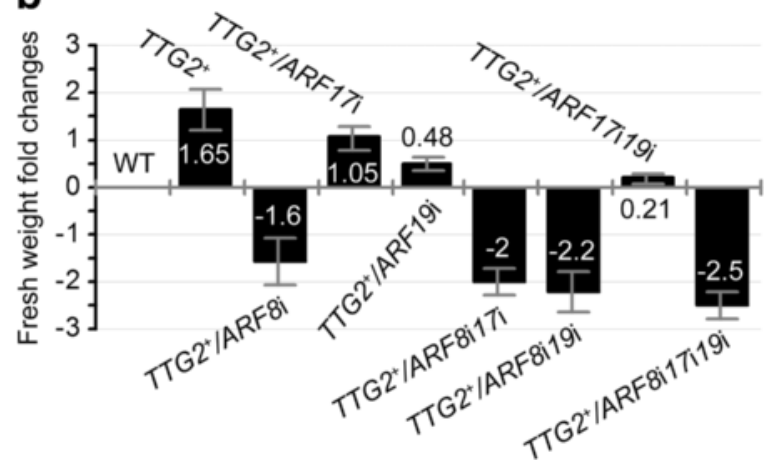

Fig. 5 Concurrent NtARF8, NtARF17, and NtARF19 silencing manipulations and the subsequent effects on plant growth. a NtARF8, NtARF17, and NtARF19 silencing efficiencies. Gene silencing was performed under $T T G 2^{+}$background to evaluate the combinational effects of NtARF8, NtARF17, and NtNAR19 on the role of NtTTG2 in the plant growth. Gene expression levels were determined by RT-qPCR as transcript quantity ratios to EFla. Percent reductions of NtARF transcript quantities in the different gene silencing manipulations were quantified in relative to $T_{T G 2^{+}}$background. Gene expression levels under backgrounds of WT, TTG2 ${ }^{+}$only, and TTG2 ${ }^{+}$with a single NtARF gene silencing are shown for comparisons of plant growth changes. b Plant fresh weight alterations by NtTTG2 overexpression and NtARF silencing compared to WT background. Data shown in $\mathbf{a}, \mathbf{b}$ are mean values \pm SEM bars ( $n=6$ experimental replicates)

plant growth enhancements, as indicated by the independent and concurrent overexpression of NtARF8 and NtTTG2 (Additional file 5: Figure S4).

We compared an $A R F 8^{+}: Y F P$ line and a TTG2 $2^{+}: R F P /$ $A R F 8^{+}: Y F P$ line with WT:RFP, TTG2i, NtARF8i, TTG2i/ $N t A R F 8 \mathrm{i}$, and $T T G 2^{+}: R F P$ plants in terms of seed production quantity. Although seed densities in fruits were similarly observed in some genotypes (Fig. 6d), the number of seeds in a single fruit significantly varied with genetic modification of NtTTG2 and NtARF8 (Fig. 6e). Independent silencing of NtTTG2 and NtARF8 resulted in acute seed abortion, whereas more than $97 \%$ and $96 \%$ seeds were aborted (Fig. 6e), respectively, in $N t T T G 2 \mathrm{i}$ and NtARF8i plants compared to that observed in WT:RFP. More severe seed abortion resulted from concurrent NtTTG2 and NtARF8 silencing, which further reduced seed number per fruit over the single gene silencing effects (Fig. 6e). Seed abortion lead to shrunken fruits, which is as an evident phenotype that was associated with NtTTG2 and NtARF8 silencing compared to the observed normal seed appearance with WT:RFP (Fig. 6d). In contrast, the independent overexpression of NtTTG2 or NtARF8 substantially promoted seed production, causing a $45 \%$ and $32 \%$ increase in seed numbers in single fruits (Fig. 6e), along with the formation of plump fruits (Fig. 6d). An enhancement of seed production was observed with concurrent NtTTG2 and NtARF8 overexpression, which resulted in an $11 \%$ and $24 \%$ increase in seed number compared to the effects of the independent overexpression of NtTTG2 and NtARF8, respectively (Fig. 6e). These findings suggest that NtARF8 is an essential component of NtTTG2regulated seed production in tobacco. This notion is in agreement with the altered NtTTG2 expression in young fruits (Additional file 4: Figure S3) and additionally confirms the role of NtTTG2 in seed production [19].

\section{NtARF8 is a functional transcription activator of illustrational target gene}

The NtARF8 protein (GenBank PMID 24875793) is the predicted protein of the NtARF8 gene, which we recently cloned from the common tobacco variety, NC89 $[4,38]$. The putative NtARF8 protein is highly identical at the amino acid level to well-characterized homologs (Additional file 6: Figure S5) and shares the conserved $\mathrm{Q}$ tract at the middle region (Fig. 7a), which characterizes ARF transcription activators [27, 31]. The Q-rich region spans residue sites 348 to 598 in the NtARF8 sequence (Fig. 7a), which consists of 843 amino acids in total. Based on these structural characteristics, NtARF8 is a potential transcription activator.

To validate this prediction, we used the GH3 (Nt-gh3) gene to illustrate the role of NtARF8 in transcriptional activation. The rationale behind this experiment is that GH3 is an auxin-responsive gene in tobacco $[22,40]$ and was recently detected in the NtTTG2-regulated profile of the tobacco transcriptome [19]. GH3 expression is NtTTG2-dependent, enhanced by NtTTG2 overexpression, but repressed by NtTTG2 silencing, whereas NtARF8 performs as an NtTTG2 gene, affecting GH3 expression (Additional file 7: Figure S6). Moreover, typical 

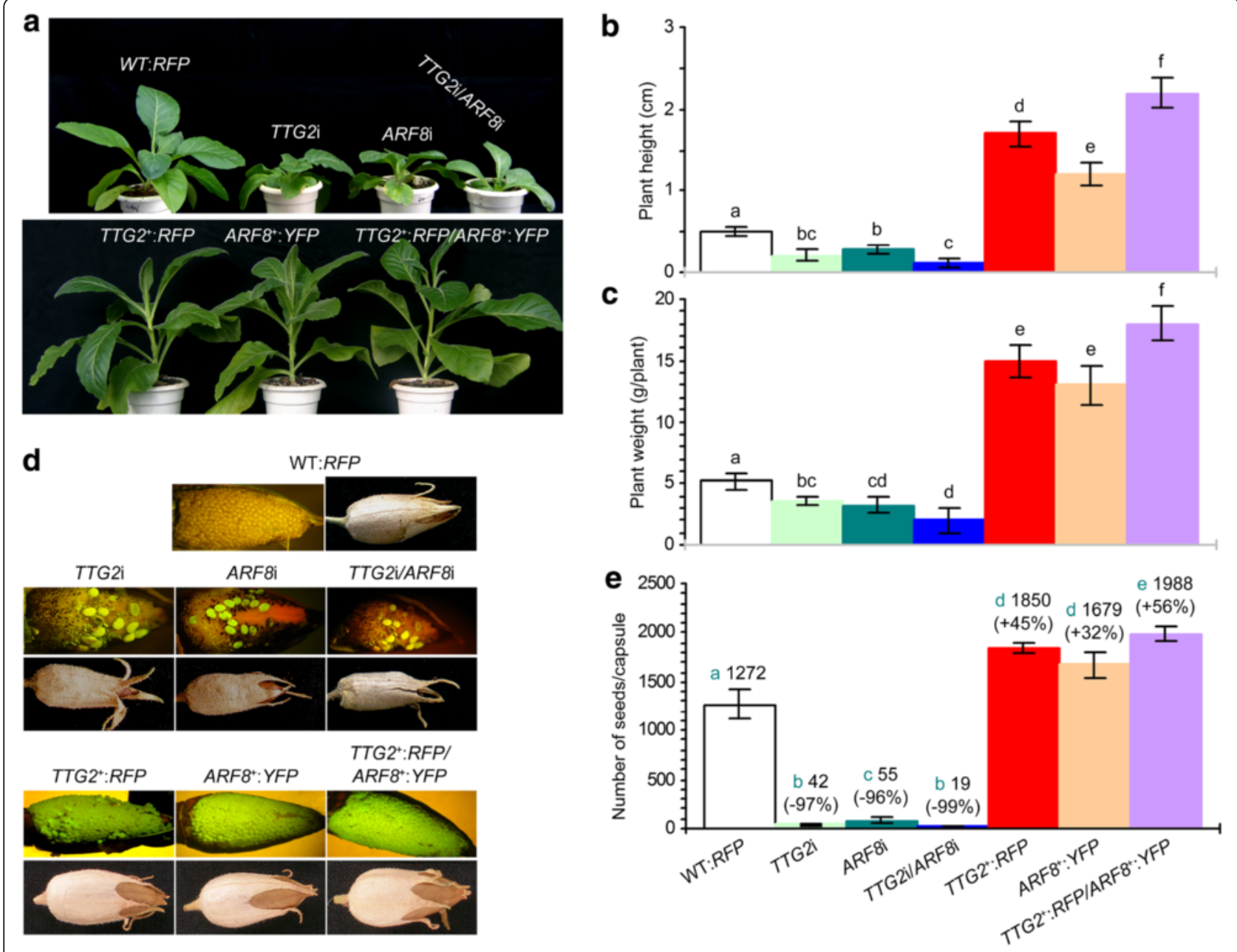

Fig. 6 The effects of concurrent TTG2 and ARF8 modifications on plant growth and seed production. Plants were grown in the greenhouse (plants used in other experiments were grown in the chamber). a Appearance of 50-day-old plants. b Plant height scoring. $\mathbf{c}$, $\mathbf{d}$ Seed production and fruit appearance. Data shown in $\mathbf{b}, \mathbf{c}$, e are means \pm SEM bars; different letters indicate significant differences among plant genotypes $(n=6 ; P<0.01)$

auxin response element TGTCT [26] exists as two repeats (409-413 and 798-802) in the GH3 promoter (GenBank Accession number AJ620494). In an electrophoresis mobility shift assay (EMSA), this element prepared as a WT probe was shown to bind to the NtARF8-His fusion protein, but not the mutant ACAGC (Fig. 7b).

Chromatin immunoprecipitation (ChIP) using chromatin isolated from WT:RFP, NtARF8i, and NtARF8 ${ }^{+}$:YFP leaves was performed to test whether NtARF8 could bind to the GH3 promoter. The specific RFP antibody was used to identify the target DNA composition of the chromatin sample, and the target DNA was assumed to combine with the ARF8-RFP fusion protein present in the same chromatin sample. The precipitated DNA was quantified by PCR in parallel with two pertinent controls. One was the input reference, in which a part of the chromatin sample was not subjected to immunoprecipitation. Another was the antibody-absent reference $(-A B)$, in which a different part of the chromatin sample was subjected to the standard immunoprecipitation procedure in the absence of the antibody. These DNA samples were PCR amplified using primers that were specific to the promoter or coding sequence (CDS) of the GH3 gene. Figure 7c shows that no GH3 CDS DNA was detected in the chromatin of the three plants, whereas the GH3 promoter was detected only in the $N t A R F 8^{+}$:YFP chromatin samples, thereby suggesting the specific binding of NtARF8 to the promoter. Similarly, the GH3 gene was expressed in $A R F 8^{+}: Y F P$ or WT:RFP, but not in $A R F 8 \mathrm{i}$ plants, and the gene transcript was 5-fold higher in $A R F 8^{+}$:YFP plants than in WT:RFP plants (Fig. 7d). These findings indicated that NtARF8 indeed is a transcription activator of the illustrational target gene. 
a 348'-QPRVSLWEIEPLTTFPMYPSLFPLRLKRPWYPGTSSF QENNSETINGMAWLRGEGGDQGPHLMNLQSFGMLPWMQ QRVDPTILRNDLNQQYQAMLATGLQNFGSGDMLKQQLMQ FQQPVQYLQHAGSHNPLLQQQQQQQAMQQQQAIHQHML PAQTQMDNVQRQPQQQVGNQMDDQAHQHSYQEAYQISH SQLQQKQPSNIPSQSFSKPDFADPNSKFAASIAPSVIPMGL GSLCSEGSSNFLNFNRIGQQ-598'

b

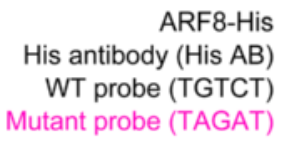

$+\quad+$

$++$

$+$

Mutant probe (TAGAT)

Immunoprecipated complex $($ ARF8-His + WT probe + His AB) $\rightarrow$

Probe $\rightarrow$

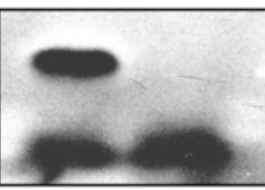

C
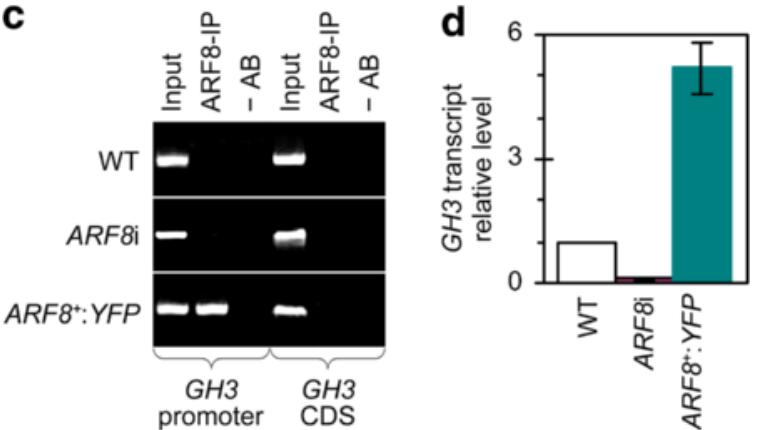

Fig. 7 Partial sequence characterization and assays of the NtARF8 protein as a transcription activator. a Partial sequence of the predicted NtARF8 protein. The NtARF8 sequence contains 843 amino acids and only the middle region is presented to indicate glutamine (Q) abundance as the characteristics of ARF transcription activators. b An EMSA assay of the NtARF8-His (ARF8-His) fusion protein, the WT probe for the auxin-responsive TGTCT element, which is present in the GH3 gene promoter, and the mutant probe. c ChIP PCR conducted with chromatins isolated from leaves of plants indicated on left, the specific YFP antibody, and primers specific to the promoter and CDS of GH3. Bands in gel indicate products of PCR conducted with DNA templates from the precipitated complex consisting of chromatin fragments and the NtARF8-YFP fusion protein (ARF8-IP), the mock precipitation in the absence of the antibody $(-A B)$, and chromatins before immunoprecipitation (input), respectively. d GH3 expression levels quantified by RT-qPCR. The WT transcript quantity ratio of GH3 to EFla was defined as 1 and scored accordingly for ARF8i and $A R F 8^{+}:$YFP. Data shown are mean values \pm SEM bars $(n=3$ experimental replicates)

\section{NtTTG2 facilitates nuclear localization of NtARF8}

To infer how NtTTG2 regulates the function of NtARF8 as a transcription activator, we tested whether both proteins directly interacted. No interaction was detected by using a yeast two-hybrid assay or by bimolecular fluorescence complementation (Additional file 8: Figure S7). Then, we monitored the subcellular distributions of both NtTTG2-RFP and NtARF8-YFP fusion proteins after transfecting the roots of WT:RFP, TTG2i, and TTG2 ${ }^{+}: R F P$ plants with the NtARF8:YFP fusion gene. Fluorescence imaging of transfected root cells indicated that the subcellular localization of NtARF8 changed with NtTTG2 quantity alterations (Fig. 8a). Fluorescence was absent under the TTG2i background, whereas high levels of RFP fluorescence was observed in the cytoplasm and nucleus of the WT:RFP cells. Under the TTG2 $2^{+}$:RFP background, strong fluorescence of the NtTTG2-RFP fusion protein was observed in both the cytoplasm and nucleus (Fig. 8a). The three genetic backgrounds had different impacts on the subcellular localization of the NtARF8-YFP fusion protein. This protein was observed with TTG2i, at a high level of accumulation in the WT:RFP and $T T G 2^{+}: R F P$ roots (Fig. 8a). Similar to the dual localization of NtTTG2, NtARF8-YFP was also detected in both the cytoplasm and nucleus of WT:RFP cells. In TTG2 $2^{+}$:RFP, NtARF8-YFP was predominantly detected in the nucleus (Fig. 8a).

To quantify the effect of altered NtTTG2 levels on the subcellular localization of NtARF8, we counted the number of root cells showing fluorescence of NtTTG2RFP (Fig. 8b) or NtARF8-YFP (Fig. 8c) only in the cytoplasm, only in the nucleus, and simultaneously in both. Our observations confirmed the dual localization of NtTTG2-RFP in both the cytoplasm and nucleus (Fig. 8c). The number of cells with cytoplasmic, nuclear, or dual location of NtARF8-YFP was determined by quantities of NtTTG2 under the different genetic backgrounds (Fig. 8c). NtARF8-YFP was detected only in the cytoplasm and only in the nuclei at approximately equivalent proportions (40\%-50\%) of WT:RFP cells, whereas NtARF8-YFP was exclusively localized to nuclei in more than $95 \%$ of the $T T G 2^{+}$:RFP cells. In both cytoplasmic and nuclear spaces, NtARF8-YFP was observed at small amounts or was not detected under the TTG2i background (Fig. 8c). These data suggest that overexpressed NtTTG2 promoted NtAFR8 localization to the nucleus.

\section{NtTTG2-facilitated nuclear localization of NtARF8 enhances its role as a transcriptional activator} The results of root cell fluorescence imaging (Fig. 8a-c) were in agreement with the findings of immunoblotting analysis of proteins from cytoplasmic and nuclear fractions of leaf cells (Fig. 8d). Cytoplasmic and nuclear proteins were analyzed together with phosphoenolpyruvate carboxylase (PEPC) and histone H3, which were used as cytoplasmic and nuclear markers, respectively [4, 41]. A group of protein blots were hybridized with the NtTTG2 antibody, which was generated by immunizing a New Zealand white rabbit in our previous study [4]. This hybridization detected remarkable quantities of the NtTTG2 protein in WT:RFP and $T T G 2^{+}$:RFP plants, although a low protein expression level was observed with 


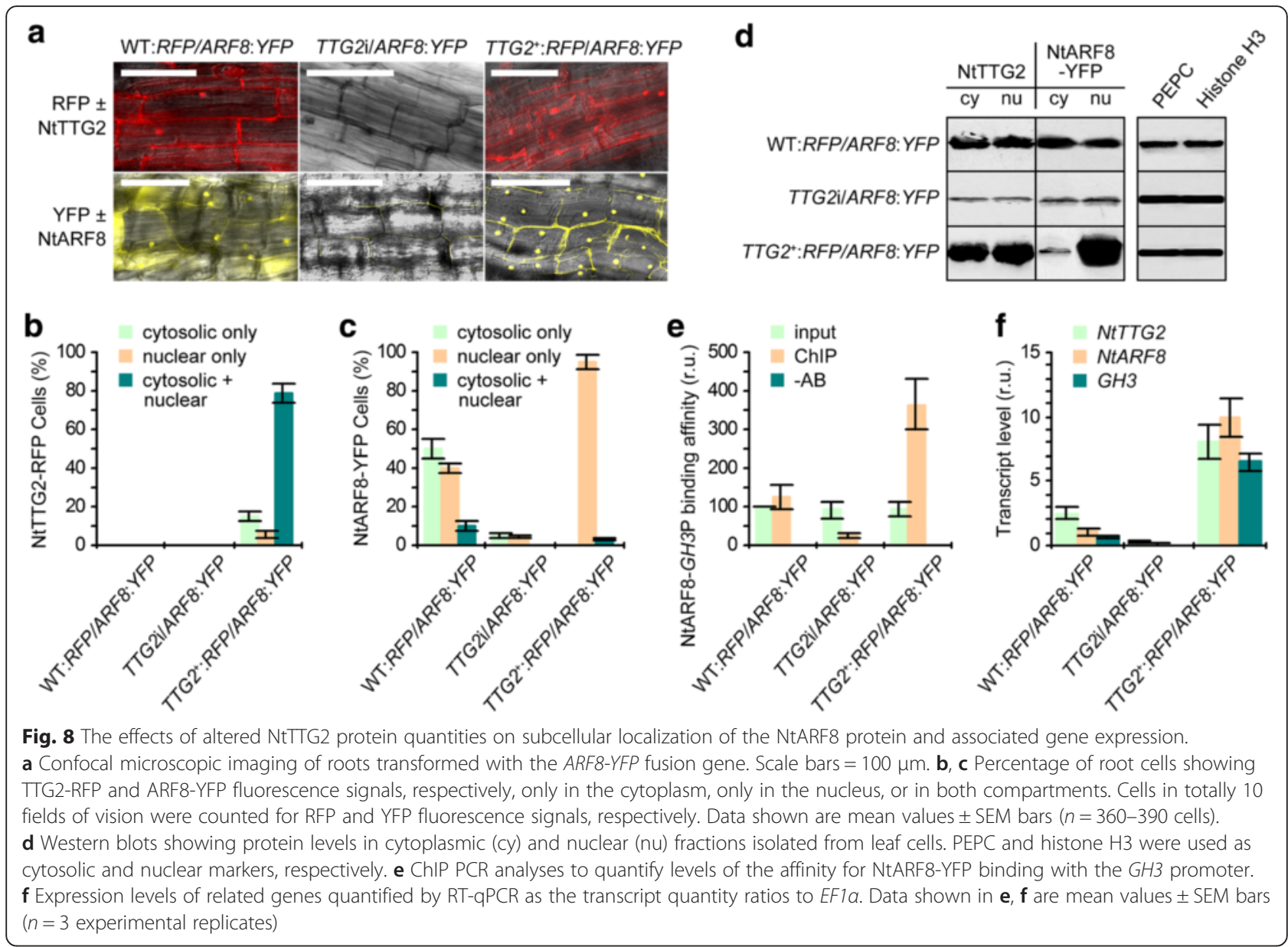

TTG2i. While similar levels of NtTTG2 were observed in the cytoplasmic and nuclear fractions of WT:RFP, a higher level was observed in the nucleus compared to that detected in the cytoplasm of $T T G 2^{+}: R F P$ cells (Fig. 8d). An independent group of protein blots were hybridized with a specific commercially manufactured YFP antibody [4]. Hybridization showed that the NtARF8YFP fusion protein was expressed at similar amounts in the cytoplasm and nucleus of WT:RFP cells, whereas it was downregulated in TTG2i cells. In $T T G 2^{+}$:RFP cells, a high level of NtARF8-YFP was detected in the nucleus, whereas a lower level was observed in the cytoplasm (Fig. 8d). These findings confirmed that overexpressed NtTTG2 facilitates in the localization of NtARF8 to the nucleus. These results also suggest that sufficient production and nuclear localization of NtTTG2 are required for the nuclear localization of NtARF8.

Nuclear localization is a prerequisite for transcription factors to function in gene expression regulation [42]. Thus, it is logical to hypothesize that NtTTG2-modulated subcellular localization of NtARF8 is mandatory to affect the expression of the GH3 gene, which was used as an illustrational target of NtARF8 (Fig. 7b-d).
A possible intermediate mechanism is that increased nuclear localization offers more opportunities for the transcription factor to bind to the target promoter. This hypothesis was validated by the ChIP PCR assay and by quantification of gene expression levels in leaves. A foliar ChIP DNA sample was quantified by RT-qPCR in parallel with a DNA sample from the input reference and another DNA sample from the $A B$ absent reference. By using this elaborate assay, the affinity of NtARF8-YFP binding with the GH3 promoter (GH3P) was quantified as relative amounts of PCR product from quantitative leaf samples that were processed in a uniform reaction volume. Figure 8e shows that a very low degree of ARF8-GH3P binding affinity was detected with TTG2i/ARF8:YFP compared to the reference level from input. Inversely, ARF8-GH3P binding affinity significantly increased with $T T G 2^{+}: R F P / A R F 8: Y F P$ compared to that observed with WT:RFP/ARF8:YFP background (Fig. 8e). These findings indicate that NtTTG2facilitated nuclear localization of NtARF8 increases its ability to bind with the GH3 promoter. GH3 was thus upregulated in $T T G 2^{+}: R F P$ leaves, but its expression was highly repressed in TTG2i:RFP compared to the 
observed steady-state expression in leaves of WT:RFP plants (Fig. 8f). A similar pattern of foliar expression was observed with NtARF8 and NtTTG2 (Fig. 8f). Therefore, NtARF8 and NtTTG2 involve a concerted regulatory mechanism at the transcription level in addition to its concomitant role in subcellular localization.

\section{NtTTG2 and NtARF8 coordinately respond to auxin}

The functional coordination of NtTTG2 and NtARF8 was further evidenced by the concomitant expression of both genes under two circumstances. First, NtTTG2 silencing and overexpression resulted in a significant enhancement and repression of NtARF8 expression, respectively, whereas similar modifications of NtARF8 did not affect NtTTG2 expression (Additional file 7: Figure S6). Therefore, NtTTG2 is indispensable to NtARF8 expression, implying that NtTTG2 functions upstream of $N t A R F 8$ in regulating the plant growth and development. Second, NtARF8 resembled NtTTG2 in terms of its response to auxin and expression enhancement in $60 \mathrm{~h}$ after NAA application to the WT plant (Fig. 9). In a parallel experiment, the amount of NtARF8 transcripts increased by 6.9 times at $60 \mathrm{~h}$ after the NAA treatment compared to the control. At the same time point, NtTTG2 expression was upregulated 5.3 times compared to the steady-state levels in detected in the control (Fig. 9). The coordinate responses of NtTTG2 and NtARF8 to exogenously applied NAA suggest that both genes are concurrently involved in auxin signaling.

\section{Discussion}

Previous studies have shown that TTGs regulate plant developmental processes and/or immune responses by interacting or cooperating with different functional partners $[4,10,12,18]$. In the Arabidopsis trichome developmental model, the functional partners of AtTTG1 include two distinct transcription factors, a bHLH [12] and a MYB [18]. In tobacco, NtTTG1 regulates immune responses by recognizing a biotic elicitor and involving transcription factor, NPR1 [10]. In contrast, NtTTG2 is characteristic of an immunity repressor, which particularly suppresses the NPR1 functional pathway to confer disease susceptibility [4]. NtTTG2 also acts as a positive regulator of development and functions in association with auxin signaling, particularly in relation to the differential expression of $12 N t A R F$ genes [19]. In the present study, we elucidated a previously unappreciated mechanism by which NtTTG2 regulates vegetative growth and seed production. This novel mechanism is executed by the genetic cooperation of NtARF8, NtARF17, and NtARF19, which are abundantly expressed in the NtTTG2upregulated transcriptional profile of auxin-related genes [19] (Additional file 2: Table S1). We further elucidated that NtARF8 plays a predominant cooperative role with NtARF17 and NtARF19 that influences the NtTTG2 functional pathway.

We have shown that ARF8 is a major mechanistic linker between NtTTG2 and auxin signaling in the regulation of tobacco growth and development. Evidence has

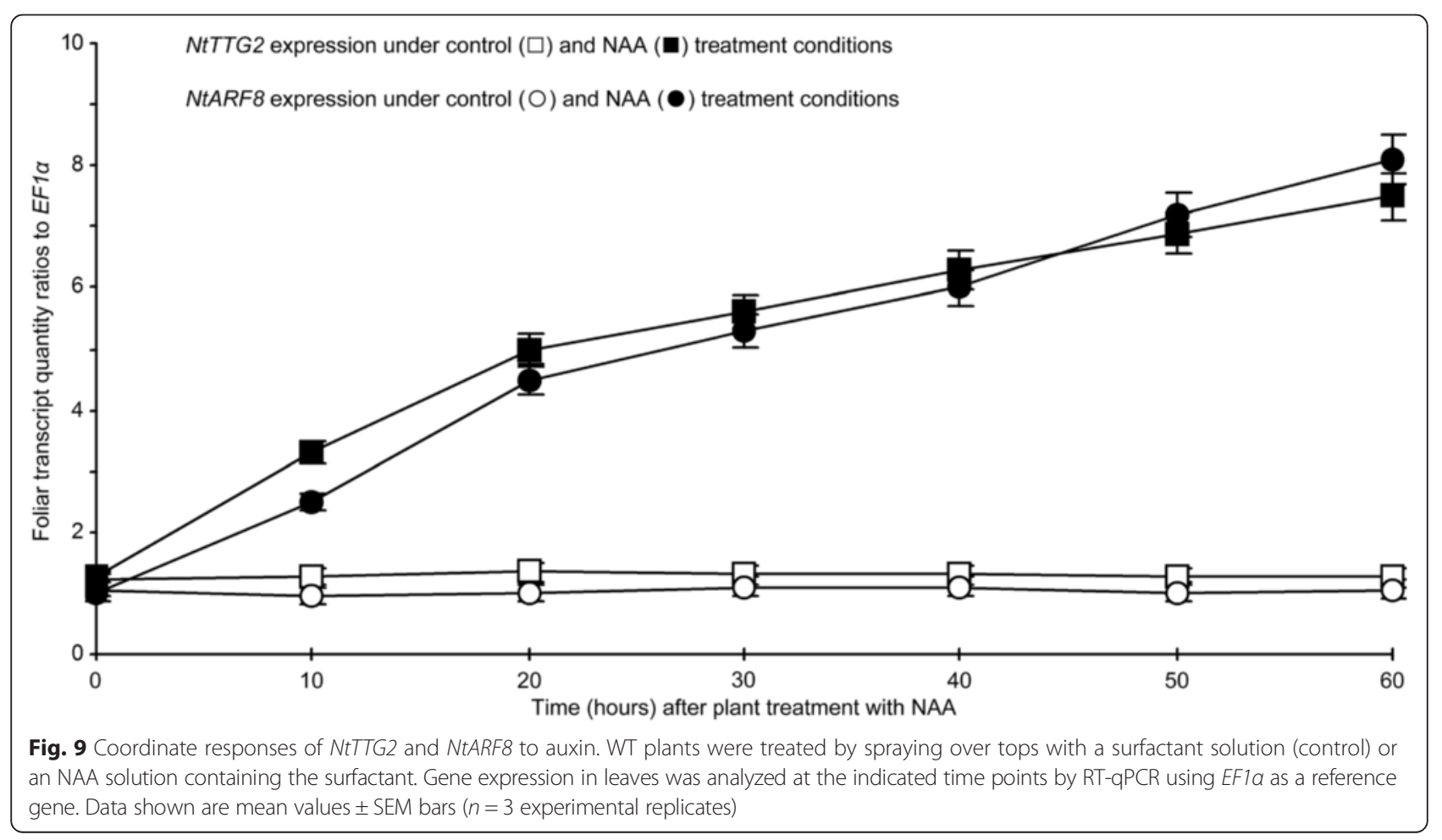


been provided at the transcriptional and posttranscriptional levels, as well as the cytological scale, particularly with respect to the functional relationship between NtTTG2 and NtARF8 genes and proteins. At the transcriptional level, the NtTTG2 gene is induced by the exogenously applied auxin (Fig. 1), but alterations in the expression levels of NtTTG2 does not affect the endogenous content of auxin in different plant organs (Additional file 1: Figure S1). Based on the link between NtTTG2 and auxin signaling, the NtARF8 gene appears to be the most possible candidate. NtARF8 is one of newly characterized 12 NtTTG2-regulated NtARF genes (Additional file 2: Table S1) that were originally identified as 13 putative $A R F$ genes in the tobacco transcriptome [19]. The expression of NtARF8 (comp30272_c0 and comp42904_c0) was upregulated by NtTTG2 overexpression and downregulated by NtTTG2 silencing. Under the same conditions, NtARF17 (comp39443_c0) and NtARF19 (comp38146_c2) showed a similar behavior as that of $N t A R F 8$, with lower levels of expression than NtARF8 but higher than the additional 9 NtTTG2regulated $A R F$ genes [19]. These findings suggest that NtTTG2 acts after auxin production to regulate $A R F$ gene expression.

Based on the gene silencing effects, 10 and 2 of the 12 characterized $A R F$ genes were respectively positively and negatively regulated NtTTG2 (Fig. 2). In the NtTTG2upregulated genes, NtARF8 silencing was more effective than silencing NtARF17 or NtARF19 to repress the growth and to eradicate the enhanced growth effect of NtTTG2 overexpression (Figs. 3, 4 and 5). This finding is suggestive of the dominant role of NtARF8 in relation to NtARF17 and NtARF19. By integrating these findings with those of the gene silencing effects on seed production quantity (Fig. 6), we propose that NtARF8 is an integral component of NtTTG2-regulated plant growth and development. At the molecular level, the functional relationship between NtTTG2 and NtARF8 differs from that of the WD40-bHLH-MYB complex for the trichome development $[12,19]$, and is also distinct from that of the AtARF8-bHLH interaction for petal growth [8] in Arabidopsis. At the phenotypic level, the functional pathway consisting of NtTTG2 and NtARF8 is responsible for vegetative growth and seed production, but whether it also affects other NtTTG2-regulated traits [4, 19] remains to be determined.

At the cytological level, the NtTTG2 protein indirectly regulates the function of NtARF8 as a transcription activator by modulating its localization to the nucleus (Figs. 7 and 8). The function of NtARF8 as a transcription activator has been demonstrated by using GH3, which is an NtTTG2-regulated auxin-responsive gene that was previously identified in tobacco $[16,23]$ (Additional file 7: Figure S6) as an NtARF8 potential target genes. The rationale behind the selection of the tobacco GH3 gene was based on the stimulatory effect of AtARF8 on the expression of GH3 orthologs in Arabidopsis [35], namely the presence of a typical auxin response element (TGTCT) in the GH3 promoter (GenBank Accession number AJ620494). Based on element recognition by ARF transcription activators [26], the ability of the NtARF8 protein to combine with the GH3 promoter is in line with $G H 3$ expression levels that decreased by silencing but increased with NtARF8 overexpression (Fig. 7). The transcription regulatory activity of NtARF8 requires nuclear import under modulation by the NtTTG2 protein, whereas NtTTG2 fulfill this cytological role depending on its dual location in the cytoplasm and nucleus (Fig. 8). When NtTTG2 remains at the steady-state level under WT background, NtARF8 is also produced at the same steady-state level with the dual location (Fig. 8). This observation implies that the steady-state level of NtARF8 is sufficient in regulating NtTTG2-conferred development. This assumed dynamic relationship between NtTTG2 and NtARF8 is in agreement with the ready-state level of the NtTTG2 protein or the gene transcript [4] (Figs. 1 and 8) and with the basal level of auxin (Additional file 1: Figure S1) under a WT background. With overexpression, however, increased amounts of the NtTTG2 protein result in elevated proportions of NtARF8 localization to the nucleus, accompanied by t GH3 upregulation (Fig. 8). Thus, the nuclear localization of NtARF8 and its performance to activate target gene expression are essential steps in the NtTTG2 functional pathway. This pathway is executed by a crosstalk with auxin signaling, as evidenced by coordinate responses of NtTTG2 and NtARF8 to auxin (Fig. 9) and by the NtTTG2-dependent role of NtARF8 in regulating transcription of the auxin-response gene (Figs. 7 and 8).

The tobacco GH3 gene was used only as an example of an NtARF8 target gene to elucidate the function of NtARF8 as a transcription activator. We did not intend to elucidate whether GH3 contributes to NtTTG2-conferred tobacco growth and development. GH3 genes encode IAA-conjugating enzymes that presumably terminate the auxin signal for plants to retrieve auxin hypersensitivity or elicit biotic stress responses during plant growth and development [43, 44]. In Arabidopsis, AtARF8 overexpression results in enhanced $G H 3$ expression, a $30 \%$ increase of auxin content, and the development of short hypocotyls [35]. These findings indicate that the role of NtARF8 in plant growth is related to the proper level of apical dominance as a function of the auxin signal. At present, we do not have evidence that shows whether this auxin homeostasis mechanism is involved in the NtTTG2-NtARF8 functional pathway for the regulation of tobacco growth and development. 
Concomitant changes in the subcellular localization of NtTTG2 and NtARF8 are critical for NtARF8 to execute the function in the transcriptional regulation of the illustrational target gene (Fig. 8). The nuclear localization and transcription regulatory activity of NtARF8 apparently requires the upregulation of the NtARF8 protein. In this case, NtARF8 gene expression could be induced to ensure further production of the protein that is caused by NtTTG2 overexpression. Thus, nuclear localization of NtTTG2 and NtARF8 likely induces a feedback mechanism involving the transcription of the specific genes. This hypothesized feedback scheme may take place, for example, when NtARF8 executes its function on auxin-responsive genes in the presence of NPR1 to activate immune response genes. In the latter case, NPR1 is hydrolyzed after a single round of gene activation such new NPR1 molecules are used in every round of transcriptional regulation [17]. However, this proteolysis model does not exclude an alternative mechanism, i.e., overexpressed NtTTG2 transports a high amount of NtARF8 into the nucleus, where NtARF8 functions to activate a large number of auxin-responsive genes such as GH3 (Fig. 8). A central question for both models is how NtARF8 is physically connected with NtTTG2. Because NtTTG2 does not directly interact with NtARF8 (Additional file 8: Figure S7), additional components that fill the apparent gap in NtTTG2-mediated nuclear import of NtARF8 should be identified. The candidates are most likely to be particular members of the impotin protein family as these proteins function as cargo carriers for the nucleocytoplasmic trafficking of proteins and nucleic acids [45]. Unknown mediators such as importins may intercede the linkage between NtTTG2 and NtARF8, and assist NtTTG2 in transporting NtARF8 into the nucleus. Testing of this hypothesis will be the subject of future studies.

\section{Conclusions}

Genetic analyses indicate that ARF8, ARF17, and ARF19 contribute to NtTTG2-regulated tobacco growth and development, whereas ARF8 plays a major role in the process. Molecular evidence indicates that ARF8 is a functional transcriptional activator, whereas the NtTTG2 protein indirectly regulates the function of NtARF8 by modulating its localization to the nucleus. In essence, NtARF8 is an integral component of the NtTTG2 functional pathway that governs the growth and development of tobacco.

\section{Methods}

\section{Plant growth and treatment}

The common tobacco variety, NC89, and transgenic lines in the T3 homozygous generation [4] were used in the present study. Transgenic plants were generated, characterized, and multiplied in this lab, and seeds of WT and transgenic plants were also maintained in this lab. For the experiments performed on roots, seeds were sterilized and germinated and subsequently, seedlings were grown on Murashige and Skoog (MS) agar medium in $10-\mathrm{cm}$ square plates under environment-controlled conditions in a plant growth chamber with a 14-h light $\left(250 \mu \mathrm{E} / \mathrm{m}^{2} / \mathrm{s}\right.$ at $\left.26{ }^{\circ} \mathrm{C}\right)$ and 10 -h dark $\left(23^{\circ} \mathrm{C}\right)$ cycle. Alternatively, seeds were sown and germinated in pots filled with a mixture of sand and potting soil, and subsequently seedlings were grown in the plant growth chamber. At 30 days after seed germination, seedlings were transferred into new pots containing the same substrate and grown under different conditions depending on the experimental purposes. Plants used in the evaluation of vegetative growth were grown in the chamber, and those used for seed production were grown in a greenhouse at $23{ }^{\circ} \mathrm{C}-26{ }^{\circ} \mathrm{C}$. After continuous growth in the chamber for an additional 20 days, plants were used in the gene silencing experiments or treated for analysis of NAAinduced NtTTG2 expression. NAA was prepared as a $2 \mu \mathrm{M}$ aqueous solution amended with $0.02 \%(\mathrm{v} / \mathrm{v})$ of surfactant Silwet-77 and applied by spraying over plant tops with an atomizer. A $0.02 \%$ Silwet-77 solution was similarly applied to controls. The treated plants were used in the analysis of gene expression at a 10-h interval in $60 \mathrm{~h}$ after treatment.

\section{Gene expression analysis}

Depending on the study purpose, RNA was isolated from intact roots, top 5-cm stems, the top sixth leaves, S3 stage flowers [19], and 15-day-old immature fruits of 70-day-old plants. Northern blots were hybridized to specific probes labeled with digoxigenin [46]. RT-PCR and RT-qPCR were conducted using specific primers (Additional file 9: Table S2) and previously described protocols [47]. The constitutively expressed EF1 $\alpha$ gene $[47,48]$ was used as reference. The relative expression level of each tested gene was quantified by RT-qPCR using that of the EF1 $\alpha$ gene as reference [48].

\section{Endogenous auxin measurements}

Endogenous free auxin (IAA) concentrations were determined by using a previously described protocol [49]. To isolate free IAA, the top sixth leaves of 30-day-old plants, S3 flowers [19], and immature fruits of 70-dayold plants were collected. Approximately $1 \mathrm{~g}$ of each organ was ground in liquid nitrogen by using a mortar and pestle. Tissue powders were dissolved in $10 \mathrm{~mL}$ of $80 \%(\mathrm{v} / \mathrm{v})$ ethanol and supplemented with $0.1 \mathrm{mg}$ of the antioxidant, 2,6-di-tert-butyl-p-cresol. The homogenate was sonicated for $30 \mathrm{~min}$ and then centrifuged $\left(4{ }^{\circ} \mathrm{C}\right.$, $10,000 \mathrm{~g}, 10 \mathrm{~min})$. The supernatant was mixed with an equal volume of mineral ether for liquid-phase 
extraction, which was repeated three times, with the bottom inorganic phase collected each time. The solution from the last extraction was adjusted to $\mathrm{pH} 8.5$ and mixed with $0.2 \mathrm{~g}$ of inylpyrrolidone, followed by three rounds of liquid-phase extraction using an equal volume of acetic ester and collection of the upper organic phase. IAA in the last organic phase was purified by chromatography using an OASIS MAX Cartridge $3 \mathrm{cc} / 60 \mathrm{mg}$ hybrid anion exchange resin (Waters Oasis). The solute was analyzed by high-performance liquid chromatography. IAA concentrations in the extracts were determined by using a standard IAA curve method. The levels of free IAA in plant organs were measured and expressed as $\mathrm{ng} / \mathrm{g}$ fresh tissue.

\section{VIGS}

Partial fragments (207-495 bp) of the NtARF genes were cloned by RT-PCR using RNA isolated from leaves and primers that were synthesized according to sequences of the corresponding unigenes (Additional file 9: Table S2). The RT-PCR products were sequenced for confirmation, and every sequence was inserted into a pBinPlus:Y35 DNA-A vector of the TCSV VIGS system [38] to generate various $A R F$-silencing constructs. Every construct was transferred into Agrobacterium tumefaciens EHA105 cells [38]. A suspension of recombinant EHA105 cells was infiltrated into the phloem of mid stems of 50-day-old plants that were grown in the chamber. A similar transfection treatment was performed using pure water in the control. Two weeks later, gene silencing efficiencies were analyzed as previously described [10].

\section{EMSA and ChIP}

EMSA and ChIP analyses were conducted by using previously described methods [47]. For EMSA, the ARF8 protein was fused to a His tag for six tandem histidine residues, and a fusion protein was produced by prokaryotic expression [48]. Previously designed WT probe, 5'CATTATTTACGTGTCTGTTTTCCTG-3', and mutant 5'-CATTATTTACGtagatGTTTTCCTG -3' [26] were used in the EMSA after labeling with biotin. The chromatin used in the ChIP assay was isolated from the top sixth leaves of 50-day-old plants as described elsewhere [50]. Complexes of chromatin fragments and NtARF8YFP (ARF8-IP complexes) were precipitated with a specific YFP antibody (Santa Cruz) and the Protein A agarose/salmon sperm DNA beads (Millipore). The NtARF8-IP complexes bound to the beads were collected by centrifugation. Concentrations of the antibody and chromatin were $10 \mu \mathrm{g}$ and $50 \mathrm{nM}$ per immunoprecipitation, respectively. Similar precipitation and centrifuge procedures were conducted in the absence of the antibody in the control. Chromatin without precipitation were used as input. DNA samples from ChIP, control, and input were analyzed separately by PCR using primers specific to the promoter and CDS of the GH3 gene (Additional file 9: Table S2). The PCR products were confirmed by sequencing.

\section{Fluorescence imaging}

The ARF8-YFP fusion gene was constructed with a plant binary vector pCAMBIA1301 that was transferred into cells of $A$. tumefaciens strain EHA105 by using a previously described method [47]. Roots of 20-day-old plants grown on MS agar medium were transformed by immersion in a suspension of the recombinant EHA105 cells. Approximately $60 \mathrm{~h}$ later, root samples were observed under a ZEISS LSM710 confocal microscope. Red and yellow fluorescence were captured at wavelengths of 591-630 and 519-560 $\mathrm{nm}$ using argon laser at excitation wavelengths of 561 and $514 \mathrm{~nm}$, respectively [10].

\section{Immunoblotting}

Total proteins were extracted from the top sixth leaves [51], the cytoplasmic fraction, and the nuclear fraction [52] of 50-day-old plants grown in the chamber. Western blots were hybridized with the specific antibody against YFP (Novagen) or the specific NtTTG2 antibody, which was produced by immunizing a New Zealand white rabbit in our previous study [47]. PEPC and histone $\mathrm{H} 3$ that were used as cytoplasmic and nuclear markers [4, 41] were hybridized with a specific PEPC antibody (Rockland) and histone $\mathrm{H} 3$ antibody (Abcam), respectively. Hybridized blots were probed by using a horseradish peroxidaseconjugated secondary antibody (Beyotime) according to the manufacturer's recommendations.

\section{Statistical analysis}

All experiments were conducted for at least three times with similar results. Quantitative data were analyzed by using the commercial IBM SPSS19.0 software package [53]. Homogeneity-of-variance in data was determined by using the Levene test, and the formal distribution pattern of the data was confirmed by using the KolmogorovSmirnov test and P-P plots. Data were subjected to ANOVA, along with Fisher's least significant difference test and Tukey-Kramer's test, respectively. Significance was tested for differences in multiple comparisons of various plant genotypes.

\section{Additional files}

Additional file 1: Figure S1. IAA concentrations in leaves, flowers, and fruits of NtTTG2-related tobacco genotypes. (PDF 45 kb)

Additional file 2: Table S1. List of newly specified NtTTG2-regulated tobacco ARF genes (PDF $18 \mathrm{~kb}$ ) 
Additional file 3: Figure S2. The chronological course of foliar NtARF8, NTARF17, and NtARF19 expression during the vegetative growth process. (PDF $40 \mathrm{~kb}$ )

Additional file 4: Figure S3. NtARF8 expression in different organs of NtTTG2-related tobacco genotypes. (PDF $50 \mathrm{~kb}$ )

Additional file 5: Figure S4. The effects of NtARF8 and NtTTG2 overexpression on plant growth. (PDF $84 \mathrm{~kb}$ )

Additional file 6: Figure S5. Alignments of selected ARF8 homologs. (PDF $342 \mathrm{~kb}$ )

Additional file 7: Figure S6. Foliar expression levels of NtTTG2, NtARF8, and GH3 under backgrounds of single and concurrent NtTTG2 and NtARF8 silencing or overexpression. (PDF $68 \mathrm{~kb}$ )

Additional file 8: Figure S7. Yeast two-hybrid and bimolecular fluorescence complementation analyses of NtTTG2 and NtARF8. (PDF $78 \mathrm{~kb}$ ) Additional file 9: Table S2. List of genes tested and primers used in this study. (PDF $48 \mathrm{~kb}$ )

\section{Abbreviations}

ARF, AUXIN RESPONSIVE FACTOR; AUXIAA, Auxin/Indole-Acetic Acid inducible; ChIP, Chromatin Immunoprecipitation; EMSA, Electrophoresis mobility shift assay; GH3, Gretchen Hagen 3; NAA, 1-naphthaleneacetic acid; NPR1, NONEXPRESSER OF PATHOGENESIS-RELATED GENES1; ParA1, An elicitin protein that is produced by an oomycete pathogen; PEPC, Phosphoenolpyruvate carboxylase; RFP, Red-fluorescent protein; RT-PCR, Reverse transcriptasepolymerase chain reaction; RT-qPCR, Quantitative real-time RT-PCR; SAUR, Small Auxin Up RNA; TCSV, Begomovirus sp. tobacco curly shoot virus; TTG, TRANSPARENT TESTA GLABRA; VIGS, Virus-induced gene silencing; YFP, Yellow-fluorescent protein

\section{Acknowledgements}

We thank Dr. Xueping Zhou for the gift of VIGS vectors.

\section{Funding}

Natural Science Foundation of China (grant number 31171830), China Novel Transgenic Organisms Breeding Project (2014ZX0800910B), and Special Public Welfare Industry Program (201303015). These funding bodies did not participate in the design of the study and collection, analysis, and interpretation of data, neither in writing the manuscript.

\section{Availability of data and materials}

Information on sequences of NtARF unigenes/candidates is provided in Additional file 2: Table S1.

\section{Authors' contributions}

$J G$ designed and performed the experiments and wrote the paper. BL designed and performed the experiments and analyzed the data. DS, JX, and $J L$ performed the experiments. HD designed the experiments and wrote the paper. All authors read and approved the final manuscript.

\section{Competing interests}

The authors declare that they have no competing interests.

\section{Consent for publication}

Not applicable.

\section{Ethics approval and consent to participate}

Not applicable.

\section{Author details}

${ }^{1}$ Plant Growth and Defense Signaling Laboratory, State Ministry of Education Key Laboratory of Integrated Management of Crop Pathogens and Insect Pests, Nanjing Agricultural University, Nanjing 210095, China. ${ }^{2}$ Yantai Academy of Agricultural Sciences, Yantai 265500, China.

Received: 7 December 2015 Accepted: 20 May 2016 Published online: 02 June 2016

\section{References}

1. Vetten N, Quattrocchio F, Mol J, Koes R. The an 11 locus controlling flower pigmentation in petunia encodes a novel WD-repeat protein conserved in yeast, plants, and animals. Gene Dev. 1997;11:1422-34.

2. Szymanski DB, Lloyd AM, Marks MD. Progress in the molecular genetic analysis of trichome initiation and morphogenesis in Arabidopsis. Trends Plant Sci. 2000;5:214-9.

3. Bouyer D, Geier F, Kragler F, Schnittger A, Pesch M, Wester K, Balkunde R, Timmer J, Fleck C, Hulskamp M.

Two-dimensional patterning by a trapping/depletion mechanism: the role of TTG1 and GL3 in Arabidopsis trichome formation. PLoS Biol. 2008;6:1166-77.

4. Li B, Gao R, Cui R, Lü B, Li X, Zhao Y, You Z, Tian S, Dong H. Tobacco TTG2 suppresses resistance to pathogens by sequestering NPR1 from the nucleus. J Cell Sci. 2012;125:4913-22.

5. Petroni $\mathrm{K}$, Tonelli $\mathrm{C}$. Recent advances on the regulation of anthocyanin synthesis in reproductive organs. Plant Sci. 2011;181:219-29.

6. Neer EJ, Schmidt CJ, Nambudripad R, Smith TF. The ancient regulatoryprotein family of WD-repeat proteins. Nature. 1994;371:297-300.

7. Biedermann S, Hellmann H. WD40 and CUL4-based E3 ligases: lubricating all aspects of life. Trends Plant Sci. 2011;16:38-46.

8. Pang $Y$, Wenger JP, Saathoff K, Peel GJ, Wen J, Huhman D, Allen SN, Tang $Y$, Cheng $X$, Tadege M, Ratet P, Mysore KS, Sumner LW, Marks MD, Dixon RA. A WD40 repeat protein from Medicago truncatula is necessary for tissuespecific anthocyanin and proanthocyanidin biosynthesis but not for trichome development. Plant Physiol. 2009;151:1114-29.

9. Stirnimann CU, Petsalaki E, Russell RB, Müller CW. WD40 proteins propel cellular networks. Trends Biochem Sci. 2010;35:565-74.

10. Wang Y, Liu R, Chen L, Liang Y, Wu X, Li B, Wu J, Wang X, Zhang C, Wang Q, Hong X, Dong H. Nicotiana tabacum TTG1 contributes to ParA1-induced signalling and cell death in leaf trichomes. J Cell Sci. 2009;122:2673-85.

11. Xu C, Min J. Structure and function of WD40 domain proteins. Protein Cell. 2011;2:202-14.

12. Morohashi K, Zhao M, Yang M, Read B, Lloyd A, Lamb R, Grotewold E. Participation of the Arabidopsis bHLH factor GL3 in trichome initiation regulatory events. Plant Physiol. 2007;145:736-46.

13. Ghanashyam C, Jain M. Role of auxin-responsive genes in biotic stress responses. Plant Signal Behav. 2009;4:846-8.

14. Truman WM, Bennett MH, Turnbull CG, Grant MR. Arabidopsis auxin mutants are compromised in systemic acquired resistance and exhibit aberrant accumulation of various indolic compounds. Plant Physiol. 2010;152:1562-73.

15. Walker AR, Davison PA, Bolognesi-Winfield AC, James CM, Srinivasan N, Blundell TL, Esch JJ, Marks MD, Gray JC. The TRANSPARENT TESTA GLABRA1 locus, which regulates trichome differentiation and anthocyanin biosynthesis in Arabidopsis, encodes a WD40 repeat protein. Plant Cell. 1999;1 1:1337-50.

16. Cao H, Glazebrook J, Clarke JD, Volko S, Dong X. The Arabidopsis NPR1 gene that controls systemic acquired resistance encodes a novel protein containing ankyrin repeats. Cell. 1997;88:57-63.

17. Spoel SH, Mou Z, Tada Y, Spivey NW, Genschik P, Dong X. Proteasomemediated turnover of the transcription coactivator NPR1 plays dual roles in regulating plant immunity. Cell. 2009;137:860-72.

18. Zhao M, Morohashi K, Hatlestad G, Grotewold E, Lloyd A. The TTG1-bHLHMYB complex controls trichome cell fate and patterning through direct targeting of regulatory loci. Development. 2008;135:1991-9.

19. Zhu Q, Li BY, Mu SY, Han B, Cui RZ, Xu MY, You ZZ, Dong HS. TTG2regulated development is related to expression of putative AUXIN RESPONSE FACTOR genes in tobacco. BMC Genomics. 2013;14:806.

20. Quint M, Gray WM. Auxin signaling. Curr Opin Plant Biol. 2006;9:448-53.

21. Vanneste S, Friml J. Auxin: a trigger for change in plant development. Cell. 2009;136:1005-16.

22. Guilfoyle TJ, Ulmasov T, Hagen G. The ARF family of transcription factors and their role in plant hormone-responsive transcription. Cell Mol Life Sci. 1998:54:619-27.

23. Liscum E, Reed JW. Genetics of Aux/IAA and ARF action in plant growth and development. Plant Mol Biol. 2002;49:387-400.

24. Kumar R, Agarwal P, Tyagi AK, Sharma AK. Genome-wide investigation and expression analysis suggest diverse roles of auxin-responsive $\mathrm{GH}$ genes during development and response to different stimuli in tomato (Solanum lycopersicum). Mol Genet Genomics. 2012;287:221-35.

25. Kumar R, Tyagi AK, Sharma AK. Genome-wide analysis of auxin response factor (ARF) gene family from tomato and analysis of their role in flower and fruit development. Mol Genet Genomics. 2011;285:245-60. 
26. Walcher $\mathrm{CL}$, Nemhauser JL. Bipartite promoter element required for auxin response. Plant Physiol. 2012;158:273-82.

27. Tiwari SB, Hagen G, Guilfoyle T. The roles of auxin response factor domains in auxin-responsive transcription. Plant Cell. 2003;15:533-43.

28. Ulmasov T, Hagen G, Guilfoyle TJ. Activation and repression of transcription by auxin-response factors. Proc Natl Acad Sci USA. 1999:96:5844-9.

29. Ulmasov T, Liu ZB, Hagen G, Guilfoyle TJ. Composite structure of auxin response elements. Plant Cell. 1995;7:1611-23.

30. Remington DL, Vision TJ, Guilfoyle TJ, Reed JW. Contrasting modes of diversification in the Aux/IAA and ARF gene families. Plant Physiol. 2004;135: $1738-52$.

31. Guilfoyle TJ, Hagen G. Auxin response factors. Curr Opin Plant Biol. 2007;10: 453-60.

32. Hagen G, Guilfoyle T. Auxin-responsive gene expression: genes, promoters and regulatory factors. Plant Mol Biol. 2002;49:373-85.

33. Tromas A, Perrot-Rechenmann C. Recent progress in auxin biology. CR Biol. 2010;333:297-306.

34. Liu ZB, Ulmasov T, Shi X, Hagen G, Guilfoyle TJ. Soybean GH3 promoter contains multiple auxin-inducible elements. Plant Cell. 1994;6:645-57.

35. Heinekamp T, Strathmann A, Kuhlmann M, Froissard M, Müller A, Catherine PR, Wolfgang DL. The tobacco bZIP transcription factor BZI-1 binds the GH3 promoter in vivo and modulates auxin-induced transcription. Plant J. 2004;38:298-309.

36. Wright RC, Nemhauser JL. New tangles in the auxin signaling web. F1000prime Rep. 2015;7:19

37. Tao X, Zhou X. A modified viral satellite DNA that suppresses gene expression in plants. Plant J. 2004;38:850-60.

38. Zhao Y, Li C, Ge J, Xu M, Zhu Q, Wu T, Guo A, Xie J, Dong H. Recessive mutation identifies auxin-repressed protein ARP1, which regulates growth and disease resistance in tobacco. Mol Plant Microbe Interact. 2014;27:638-54.

39. Grabherr MG, Haas BJ, Yassour M, Levin JZ, Thompson DA, Amit I, Adiconis X, Fan L, Raychowdhury R, Zeng Q, Chen Z, Mauceli E, Hacohen N, Gnirke A, Rhind N, di Palma F, Birren BW, Nusbaum C, Lindblad-Toh K, Friedman N, Regev A. Full-length transcriptome assembly from RNA-Seq data without a reference genome. Nat Biotechnol. 2011;29:644-52.

40. Goldsbrough A, Belzile F, Yoder Jl. Complementation of the tomato anthocyanin without (aw) mutant using the dihydroflavonol 4-reductase gene. Plant Physiol. 1994;105:491-6.

41. García AV, Blanvillain-Baufumé S, Huibers RP, Wiermer M, Li G, Gobbato E, Rietz S, Parker JE. Balanced nuclear and cytoplasmic activities of EDS1 are required for a complete plant innate immune response. PLoS Path. 2010;6:e1000970.

42. Johnson CS, Kolevski B, Smyth DR. TRANSPARENT TESTA GLABRA2, a trichome and seed coat development gene of Arabidopsis, encodes a WRKY transcription factor. Plant Cell. 2002;14:1359-75.

43. Mittag J, Gabrielyan A, Ludwig-Müller J. Knockout of GH3 genes in the moss Physcomitrella patens leads to increased IAA levels at elevated temperature and in darkness. Plant Physiol Biochem. 2015;97:339-49.

44. Staswick PE, Serban B, Rowe M, Tiryaki I, Maldonado MT, Maldonado MC, Suza W. Characterization of an Arabidopsis enzyme family that conjugates amino acids to indole-3-acetic acid. Plant Cell. 2005;17:616-27.

45. Lü B, Su W, Li L, Dong H. Nucleocytoplasmic trafficking for regulation of defense signaling in plants. J Nanjing Agr Univ. 2011;34:129-37.

46. Peng J, Bao Z, Ren H, Wang J, Dong H. Expression of harpin $x_{0 o}$ in transgenic tobacco induces pathogen defense in the absence of hypersensitive response. Phytopathology. 2004;94:1048-55.

47. Liu R, Chen L, Jia Z, Lu B, Shi H, Shao W, Dong H. Transcription factor AtMYB44 regulates induced expression of the ETHYLENE INSENSITIVE2 gene in Arabidopsis responding to a harpin protein. Mol Plant Microbe Interact. 2011;24:377-89.

48. Chen L, Qian J, Qu S, Long J, Yin Q, Zhang C, Wu X, Sun F, Wu T, Hayes M, Beer SV, Dong $\mathrm{H}$. Identification of specific fragments of $\mathrm{HpaG}_{\text {Xooc }}$ a harpin from Xanthomonas oryzae pv. oryzicola, that induce disease resistance and enhance growth in plants. Phytopathology. 2008;98:781-91.

49. Guo X, Song C, Wang M, Zheng F, Miu W, Wang J. Determination of auxin content in tobacco and cotton by high performance liquid chromatography. Jiangsu J Agr Sci. 2012;28:225-7.

50. Benhamed M, Bertrand C, Servet C, Zhou DX. Arabidopsis GCN5, HD1, and TAF1/HAF2 interact to regulate histone acetylation required for lightresponsive gene expression. Plant Cell. 2006;18:2893-903.
51. Liu F, Liu H, Jia Q, Wu X, Guo X, Guo X, Zhang S, Song F, Dong H. The internal glycinea-rich motif and cysteine suppress several effects of the $\mathrm{HpaG}$ Xooc protein in plants. Phytopathology. 2006;96:1052-9.

52. Kinkema M, Fan W, Dong X. Nuclear localization of NPR1 is required for activation of PR gene expression. Plant Cell. 2000;2000(12):2339-50.

53. Shi LW. SPSS19.0 Statistical Analysis from Accidence to Conversance. Beijing: Tsinghua Univ Press; 2012. p. 109-43.

\section{Submit your next manuscript to BioMed Central and we will help you at every step:}

- We accept pre-submission inquiries

- Our selector tool helps you to find the most relevant journal

- We provide round the clock customer support

- Convenient online submission

- Thorough peer review

- Inclusion in PubMed and all major indexing services

- Maximum visibility for your research

Submit your manuscript at www.biomedcentral.com/submit
C Biomed Central 